\title{
Quantification and Reduction of the Residual Chemical Reactivity of Passivated Biodegradable Porous Silicon for Drug Delivery Applications
}

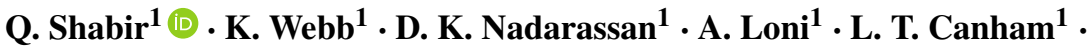 \\ M. Terracciano ${ }^{2,3} \cdot$ L. De Stefano ${ }^{2}$ I. Rea ${ }^{2}$
}

Received: 21 October 2015 / Accepted: 10 August 2016 / Published online: 7 April 2016

(C) The Author(s) 2017. This article is published with open access at Springerlink.com

\begin{abstract}
The chemical reactivity of as-anodized porous silicon is shown to have an adverse effect on a model drug (Lansoprazole) loaded into the pores. The silicon hydride surfaces can cause unwanted reactions with actives during storage or use. Techniques such as thermal oxidation or surface derivatization can lower the reactivity somewhat, by replacing the reactive silicon-hydride species with a more benign oxide or functional group. However, by using a trio of analytical techniques (fluorometric dye assay, HPLC assay, and chemography) we show that residual hydride is still likely to be present and only after combining thermal oxidation with surface derivatization can the residual reactivity be reduced to those values typically observed with sol-gel (porous) silica. Potential sources of residual reactivity are discussed, with reference to data obtained by trace metal analysis, residual solvents, and $\mathrm{pH}$ measurements.
\end{abstract}

Keywords Porous silicon · Reactivity · Derivatization . Drug delivery

Q. Shabir

qshabir@psivida.com

1 pSiMedica Ltd, Malvern Hills Science Park, Geraldine Road, Malvern, Worcester WR14 3SZ, UK

2 Institute for Microelectronics and Microsystems, National Research Council, Via P. Castellino 111, 80131, Naples, Italy

3 Department of Pharmacy, University of Naples Federico II, via D. Montesano 49, 80131, Naples, Italy

\section{Introduction}

Porous silicon has been under pre-clinical evaluation for drug delivery for a number of years [1-6]. Its medical biodegradability and biocompatibility [7-9], widely tuneable pore sizes via electrochemical etching [10,11], flexible surface chemistry $[12,13]$ and high payloads of nanostructured drugs [14] are its key attributes in this regard. However, biodegradable materials are generally, almost by definition, more chemically reactive than "bio-inert" ones. Biodegradable polymers, for example, liberate acidic byproducts that are well known to introduce local $\mathrm{pH}$ changes that can damage the tertiary structure of large biomolecules like proteins $[15,16]$. Biodegradable metal degradation products, like hydrogen gas from magnesium, may cause adverse reactions in vivo [17] and may lead to body fluid alkalization locally [18].

Biodegradable semiconducting silicon degrades into orthosilicic acid in vivo, which is naturally present in the human body [19], but can also release trace levels of silane [20] and can contain residual silicon-hydride bonds after thermal passivation (oxidation) treatments (21). If insufficient post-etch rinsing and drying is carried out the material can also contain sufficient HF-based electrolyte, solvent residues and etch by-products to cause cytotoxicity and reactivity [22]. Pharmacopeia requirements for drug formulations include very low levels of "Active Pharmaceutical Ingredient" (API) modification during long term storage (e.g. $<5 \%$ over 6 months). This necessitates modifying the vehicle or carrier if it interacts with API chemistry. In the case of mesoporous silicon, optimizing the pore wall chemistry to minimize any chemical reactions with the entrapped drug molecules is a prerequisite before loading it with active molecules. 
Porous silicon is commonly manufactured by the etching of crystalline silicon in aqueous and non-aqueous electrolytes containing hydrofluoric acid [23]. The surface of freshly prepared porous silicon is covered with a layer of $\mathrm{Si}-\mathrm{H}$ bonds with minor quantities of $\mathrm{Si}-\mathrm{F}$ and $\mathrm{Si}-\mathrm{O}$ species also present. The reactivity of as-etched porous silicon is dominated by the chemistry of $\mathrm{Si}-\mathrm{Si}$ and $\mathrm{Si}-\mathrm{H}$ bonds [12] particularly when trying to load biomolecules or actives in pores for drug delivery. As etched (as-anodized) porous silicon contains a high concentration of silicon hydride bonds at its surface; which act as a reducing agent. Therefore to make the material non-reactive with APIs, the $\mathrm{Si}-\mathrm{H}$ bonds are oxidized to yield a passivated product, which is less reactive due to lower amounts of hydrides [20,21]. Derivatization of Si-H bonds with aminosilane compounds like APTES or APDMES has also been employed to make the pore wall surface more stable and more compatible with biomolecules such as oligonucleotides [24].

In the present study, reactions of residual hydride of thermally annealed, oxide-passivated, derivatized or both oxidized and derivatized porous silicon samples were studied using three distinct assays. The first of these is a chemical method based on hydride-mediated degradation of the Lansoprazole drug, a substituted 2-(2-pyridylmethyl) sulfinyl benzimidazole molecule, used as a PPI (proton pump inhibitor) in the inhibition of gastric acid secretion. Lansoprazole shows high sensitivity to proton attack at the sulfoxide group under weak acidic conditions [25]. Silicon hydride bonds at the pore wall surface act as a reducing agent when in contact with the Lansoprazole molecule. This reducing effect on Lansoprazole is proportional to the hydride content of the porous silicon material and the drug will degrade in the reaction mixture accordingly. The percentage of degraded Lansoprazole can be used as a direct measurement of the chemical reactivity of the porous silicon.

A second method also uses a hydride-mediated oxidation reduction of phenoxazin-3-one dyes Resazurin and Resorufin [26]. In this assay, hydride from porous silicon reduces the weakly fluorescent dye Resazurin to the highly fluorescent molecule Resorufin at $\mathrm{pH} 7.6$ in phosphate buffer [27]. At the emission peak of Resorufin (587 $\mathrm{nm}$ ), fluorescence from Resazurin is negligible and is used as a blank in the assay procedure.

The third approach for reactivity assessment is chemographic analysis of the samples. Porous silicon releases low levels of gaseous silanes in humid air which create images on photographic plates through reduction of the silver halide in a photographic emulsion [28]. The low levels of monosilane emission are thought to be primarily due to hydrolysis of $\mathrm{Si}_{-} \mathrm{H}_{3}$ bonds, so relatively high concentrations of hydrides on the surface will cause the chemographic effects to be more pronounced for as-etched material than oxide-passivated or derivatized porous silicon surfaces [29].

We believe that these three methods complement each other in analyzing the residual hydrides within porous silicon post oxide-passivation or derivatization, so they were simultaneously used following a variety of chemical passivation treatments on a specific porous silicon structure, in order to quantitatively compare the resulting residual reactivity.

\section{Materials and Methods}

\subsection{Reagents}

3-aminopropyltriethoxysilane (APTES), 3-aminopropyltriethoxysilane (APDMES), 1-decene, Resazurin, Resorufin and Lansaprazole were purchased from Sigma Aldrich. All other solvents and buffers were purchased from Sigma and were research-grade chemicals.

\subsubsection{Manufacture of Porous Silicon Particles}

Porous silicon microparticles were prepared from six inch diameter $\mathrm{p}^{+}$wafers (boron-doped, resistivity range 0.005 $0.02 \Omega . \mathrm{cm}$ ) via anodization in an HF-methanol based electrolyte. Current density and time were chosen to yield two forms of free-standing mesoporous silicon layers: (a) average porosity of $86 \%$ and $227 \mu \mathrm{m}$ thickness (sample BS419) and (b) average porosity of $68 \%$ and $150 \mu \mathrm{m}$ thickness (sample BS445); after rinsing and drying, the higher-porosity samples were ball-milled at $300 \mathrm{rpm}$ in air for $1 \mathrm{~min}$, then passed through a $53 \mu \mathrm{m}$ stainless steel sieve to obtain a powder with a defined particle size distribution (Table 1); samples BS419 were subsequently oxidized at $700{ }^{\circ} \mathrm{C}$ for 16 hours in a Carbolite HTR $11 / 75$ rotary furnace and then hydrated in deionized water before drying under vacuum overnight. The lower-porosity samples (BS445) were rotor-milled at 20,000 rpm to yield powder with a size distribution and gas adsorption properties shown in Table 1.

\subsubsection{Characterization of Porous Silicon Particles}

Porous silicon particle size was measured using the Mastersizer 2000 laser diffraction system with a Hydro MU dispersion unit. The material was wet to a paste with $5 \%$ (w/w) Igepal CA (aq) before being dispersed in deionized water for measurement. The raw scattering data was deconvolved using Mie theory and an average particle size distribution was generated from five measurements.

Gas adsorption analysis on the porous silicon powders was conducted with nitrogen and a Micromeritics TriStar 
Table 1 Physicochemical properties of oxidized porous silicon microparticles prior to derivatization treatments

\begin{tabular}{|c|c|c|}
\hline Property & Milled pSi (BS445) & Milled \& Oxidised pSi (BS419) \\
\hline Particle size distribution & $\begin{array}{l}\mathrm{d} 10=1.5 \mu \mathrm{m} ; \mathrm{d} 50=17.8 \mu \mathrm{m} \\
\mathrm{d} 90=44.2 \mu \mathrm{m}\end{array}$ & $\begin{array}{l}\mathrm{d} 10=3.9 \mu \mathrm{m} ; \mathrm{d} 50=15 \mu \mathrm{m} ; \mathrm{d} 90 \\
=43 \mu \mathrm{m}\end{array}$ \\
\hline Surface area $\left(\mathrm{m}^{2} / \mathrm{g}\right)$ & 287 & 217 \\
\hline Pore volume (ml/g) & 0.994 & 0.969 \\
\hline Pore size (APD in nm) & 13.7 & 16.6 \\
\hline $\mathrm{pH}^{\mathrm{a}}$ & Not measured & $4.1+/-0.2$ \\
\hline Residual solvents $^{\mathrm{a}}$ (ethanol/methanol) & Not measured & $<1000 \mathrm{ppm}$ \\
\hline Heavy metal impurities (class 1$)^{\mathrm{a}}$ & Not measured & None detected (XRF) \\
\hline Heavy metal impurities (class 2$)^{\mathrm{a}}$ & Not measured & $\mathrm{Cu}(17-22 \mathrm{ppm}), \mathrm{Ni}$ (11-12 ppm) \\
\hline
\end{tabular}

${ }^{\text {a }}$ Data from other batches made under nominally similar conditions
3000 system. Computational analysis of the isotherms yielded absolute values for surface area (based on the Brunauer-Emmett-Teller/BET method), pore volume (based on the Barrett-Joyner-Halenda/BJH method) and average pore diameter (APD from BJH adsorption data).

Chemical purity with respect to trace heavy metals was assessed in selected batches via X-ray fluorescence (XRF) carried out using a Bruker S8 Tiger spectrometer with a semi-quantitative software analysis suite. $1 \mathrm{~g}$ batches of material were loaded into aluminum sample cups and held in place with an ultrathin Mylar film.

Residual solvent levels (ethanol and methanol) in selected batches were quantified with a PerkinElmer Autosystem XL Gas Chromatography system with a BP624 capillary column following the pharmacopeia protocol USP/NF 467.

$\mathrm{pH}$ was determined using the pharmacopeia protocol USP/NF 791 wherein a suspension of $0.2 \mathrm{~g}+/-0.01 \mathrm{~g}$ material is sonicated for 1 minute in $5.0 \mathrm{ml}$ of carbon-dioxide free water. Measurements were conducted on a calibrated Mettler Toledo MP225 pH meter at $25+/-2{ }^{\circ} \mathrm{C}$.

FTIR was conducted on a Nicolet Continu $\mu \mathrm{m}$ XL Thermo Scientific, equipped with a microscope; measurements were performed in reflection mode on an area of 100 $\times 100 \mu \mathrm{m}^{2}$. The number of scans was set to 64 . Spectra were collected in the range of $4000-500 \mathrm{~cm}^{-1}$ with a resolution of $2 \mathrm{~cm}^{-1}$.

Surface charge measurements of bare and functionalized porous silicon nanoparticles were carried out by dynamic light scattering (DLS) using a Zetasizer Nano ZS (Malvern Instruments, Malvern, UK).

\subsubsection{Hydrosilylation of Porous Silicon Particles}

Porous silicon microparticles were placed in a Schlenk tube containing a deoxygenated neat 1-decene $(99 \% \mathrm{v} / \mathrm{v})$ and allowed to react at $110^{\circ} \mathrm{C}$ for $18 \mathrm{~h}$ under a stream of argon (Fig. 1, II, III).
Microparticles were extensively washed in tetrahydrofuran and chloroform so as to remove excess of unreacted and physisorbed reagent. Microparticles dispersion was centrifuged at each wash for $30 \mathrm{~min}$ at $13,000 \mathrm{rpm}$ and the supernatant was discarded.

\subsubsection{Silanization of Porous Silicon Particles}

The functionalization procedure is based on silane chemistry. PSi-NPs were treated with Piranha solution $\left(\mathrm{H}_{2} \mathrm{O}_{2}: \mathrm{H}_{2} \mathrm{SO}_{4} 1: 4\right)$, in order to create $\mathrm{OH}$ groups, for 30 minutes at ambient temperature. After treatment with the Piranha solution, samples were extensively washed in milli$\mathrm{Q}^{\circledR}$ water to remove any adsorbed acid (Fig. 1, I). The microparticle dispersion was centrifuged for 30 minutes at $13,000 \mathrm{rpm}$ and the supernatant was discarded. The pellet was silanized by immersion in 5\% aminosilane solution; APTES or APDMES in dry ethanol for 1 hour at room temperature. After silanization, samples were extensively washed in dry ethanol and then centrifuged for $30 \mathrm{~min}$ at $13,000 \mathrm{rpm}$ and the supernatant discarded. The pellet was incubated for 10 minutes at $100{ }^{\circ} \mathrm{C}$ (curing process).

\subsubsection{Chemical Reactivity of Porous Silicon by RP-HPLC Method}

HPLC was performed using an Agilent 1200R series with a $\mathrm{C} 18$ bonded stationary phase. The separation was achieved with an ACE C18 $(150 \times 4.6 \mathrm{~mm})$ column. Solvent A consisted of water and solvent $\mathrm{B}$ consisted of acetonitrile: water: triethylamine (80: 20: 0.005), the $\mathrm{pH}$ of solvent $\mathrm{B}$ was adjusted to 7 with orthophosphoric acid $(85 \% \mathrm{v} / \mathrm{v})$. These solutions were used to set up a gradient elution method. The mobile phase is run through the column on a linear gradient of increasing organic modifier; flow rate was set at 0.8 $\mathrm{mL} / \mathrm{min}$ and the eluent was monitored at $285 \mathrm{~nm}$.

Lansoprazole is separated from its associated degradation products according to the RP-HPLC method described 


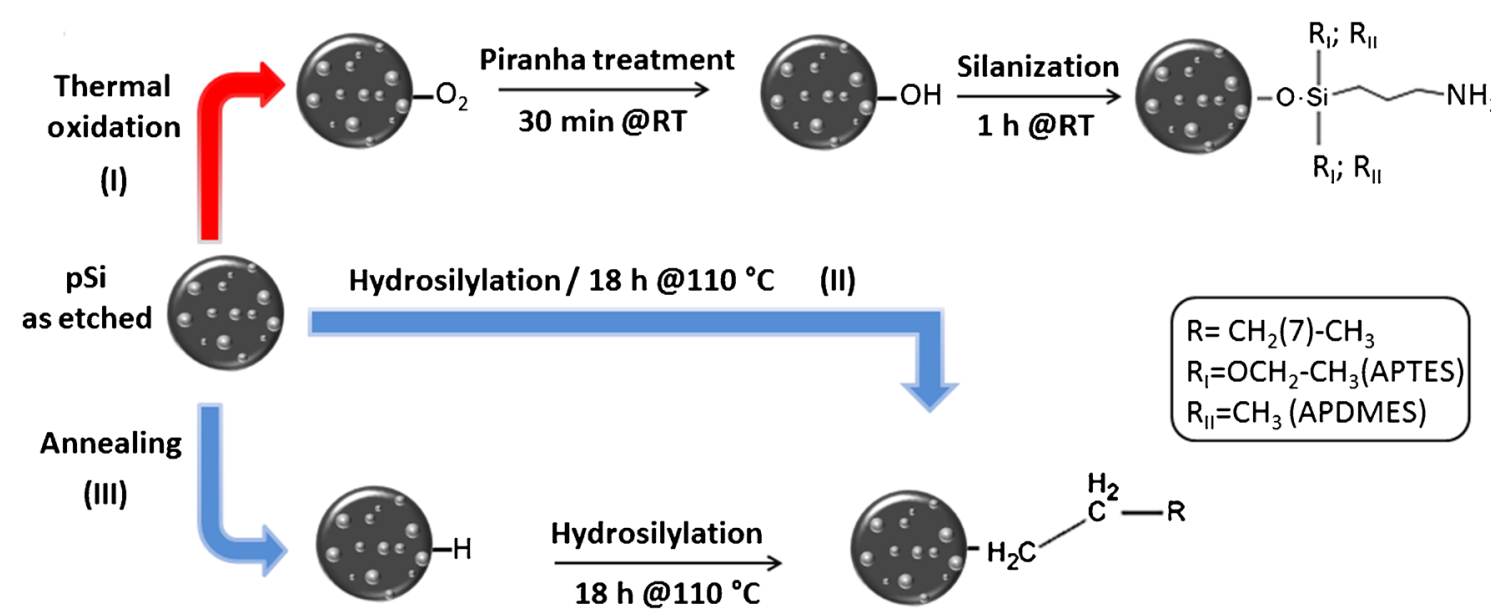

Fig. 1 Thermal and chemical passivation of porous silicon powders: (I) thermal oxidation and BS419 silanization process by APTES or APDMES; (II) BS445 chemical passivation by hydrosilylation with 1-decene; (III) BS445 annealing and hydrosilylation by 1-decene

in the United States Pharmacopeia [30] for this molecule. The Lansoprazole solution was accurately prepared at an API concentration of $10 \mathrm{mg} / \mathrm{mL}$ in methanol. Each sample required $100 \mu \mathrm{L}$ of the Lansoprazole solution. Approximately $20 \mathrm{mg}( \pm 2 \mathrm{mg}$ ) of each silicon /silica sample was weighed in duplicate into a $5 \mathrm{~mL}$ freeze drying vial. Approximately $100 \mu \mathrm{L}$ of the Lansoprazole/methanol solution was added to the samples and sealed with a butyl stopper and aluminum overseal. The samples were placed in a $25{ }^{\circ} \mathrm{C}$ water bath and incubated for 2 hours. As a control sample, $100 \mu \mathrm{L}$ of the Lansoprazole/methanol solution was pipetted into a $5 \mathrm{~mL}$ freeze drying vial and incubated alongside the other samples. As-anodized porous silicon was used as a positive control which showed maximum degradation of the drug compared with other samples.

After incubation, the overseal and the stopper were removed, taking care as the vial may contain gas as a result of any reactivity associated with the porous silicon. Each sample and the Lansoprazole control was diluted with 4.9 $\mathrm{mL}$ methanol and gently mixed. The porous silicon was allowed to settle, and then $1 \mathrm{~mL}$ of the supernatant was removed and filtered into a HPLC vial using a Whatman 0.2 $\mu \mathrm{m}$ PVDF filter.

Each sample was assayed by RP- $\operatorname{HPLC}(25,30)$ to determine the total percentage of degradation in the samples in comparison to the control (Lansoprazole in methanol) solution. The method was validated for specificity and reproducibility.

\subsubsection{Dye Reactivity by Fluorimetric Method}

A PerkinElmer 1420 Victor3 Multilevel Counter was used to quantify the highly fluorescent pink Resorufin dye which is produced by reduction of blue Resazurin by hydride surfaces of porous silicon. Fluorescence measurements utilized a CW lamp filter of $531 \mathrm{~nm}$ (excitation) and emission filter of $616 \mathrm{~nm}$ for quantification. (Alamar Blue assay, US patent No5, 501,959).

About $15 \%$ solutions of Resazurin and Resorufin were prepared in $50 \mathrm{mM}$ phosphate buffer pH7.6. From the stock solution of Resorufin various dilutions were made in phosphate buffer (pH7.6) to achieve a sufficient number of solutions within the concentration range $0-2.0 \mu \mathrm{g} / \mathrm{mL}$ for the generation of a standard calibration plot.

From the $15 \%$ solution of Resazurin, a $2.5 \mu \mathrm{g} / \mathrm{mL}$ working solution was prepared in the same phosphate buffer (pH7.6). Duplicate samples of $20 \pm 0.5 \mathrm{mg} \mathrm{pSi}$ were weighed into amber Eppendorf tubes. About $1 \mathrm{~mL}$ of the $2.5 \mu \mathrm{g} / \mathrm{mL}$ working Resazurin dye solution was added to each silica/silicon sample to give a suspension of $20 \mathrm{mg} / \mathrm{mL}$. After two hours of incubation at $25^{\circ} \mathrm{C}$, the Eppendorf tubes were centrifuged at $14000 \mathrm{rpm}$ for 2 minutes and $200 \mu \mathrm{L}$ of the supernatant liquid was transferred to a 96 well plate in triplicate. The fluorescence values of each sample were compared to that of the blue Resazurin dye solution, to that of silica which has no hydride bonding and to that of $500{ }^{\circ} \mathrm{C}$ oxidized pSi which has a significant number of hydrides but still can be wetted with the buffer.

\subsubsection{Chemographic Method:}

Chemography utilized $25 \mu \mathrm{m}$ thick Ilford L4 nuclear tracking emulsion plates with a custom built light-tight box wherein there was $100 \%$ humidity at $25^{\circ} \mathrm{C}$.

Porous silicon powders $(20 \mathrm{mg})$ which were as-etched, oxide passivated or derivatized were weighed and then placed in direct contact with the nuclear emulsion plate in air of $100 \%$ relative humidity at $25{ }^{\circ} \mathrm{C}$ for times ranging 
from 10 minutes to 100 hours in complete darkness. The position of the powders was recorded by photography prior to plate exposure. After exposure, the plates were soaked in Ilford developer for 2 minutes, soaked in Ilford stop solution for 1 minute and fixed in Ilford fixer for 15 minutes. All of these operations were conducted at $25^{\circ} \mathrm{C}$. Subsequent plate washing in stream of water for 15 minutes was again carried out at room temperature.

\subsection{Results}

\subsubsection{Derivatization of pSi Particles}

Porous silicon particles surface chemistry has been modified in three different ways, sketched in Fig. 1: the first route is a three-step procedure which starts with a thermal oxidation in wet atmosphere at $400{ }^{\circ} \mathrm{C}$, followed by an activation in Piranha solution and a silanization of powders that leads to an amino-terminated surface; the second is a direct passivation of the $\mathrm{pSi}$ surface in pure 1-decene which gives a $\mathrm{Si}-\mathrm{C}$ surface; the third is thermal annealing in inert atmosphere at $400{ }^{\circ} \mathrm{C}$ plus hydrosilylation, having the same result as route (II).

The passivation treatments of $\mathrm{pSi}$ powders were analysed by FTIR spectroscopy. In Fig. 2 and Table 2 the spectra changes of $\mathrm{SiH}$ and annealed $\mathrm{SiH}$ powders after thermal hydrosylilation by 1-decene are reported: the comparison between FTIR spectra before and after treatments, confirmed an almost complete conversion of $\mathrm{pSi}$ particle surface chemistry. The evidence is the presence of characteristic peaks of $\mathrm{Si}-\mathrm{C}$ covalent bond: stretching modes of $\mathrm{C}-\mathrm{H}_{x}$ bonds at 2957-2853, 1465-1368 and $725-720 \mathrm{~cm}^{-1}$. However, all FTIR spectra showed a weak characteristic peak of backbone $\mathrm{Si}-\mathrm{H}$ bonds at $2100 \mathrm{~cm}^{-1}$ and the peak of
Table 2 FTIR peak values of porous silicon powders (BS419) after hydrosilylation process

\begin{tabular}{|c|}
\hline $\begin{array}{c}\mathrm{SiH} / \mathrm{Hydrosilylated} \mathrm{SiH} \\
\text { Annealed } \mathrm{SiH} / \mathrm{Hydrosilylated} \mathrm{SiH}\end{array}$ \\
\hline a $1=2957-2853 \mathrm{~cm}^{-1} \mathrm{CHx}$ asymmetric stretch \\
\hline a $2=1456-1368 \mathrm{~cm}^{-1} \mathrm{CHx}$ symmetric stretch \\
\hline a $3=725-720 \mathrm{~cm}^{-1} \mathrm{CH}$ stretch \\
\hline b $=2100 \mathrm{~cm}^{-1} \mathrm{Si}-\mathrm{H}$ stretch \\
\hline $\mathrm{c}=1090-1100 \mathrm{~cm}^{-1}$ Si-O-Si stretch \\
\hline
\end{tabular}

Si-O-Si stretching mode at $1090-1100 \mathrm{~cm}^{-1}$ probably due to spontaneous ageing of $\mathrm{pSi}$ powders during the handling.

FTIR spectra of $\mathrm{SiH}$ and $\mathrm{SiO}_{2}$ powders, in Fig. 3 and Table 3, confirmed the success of silanization: both samples showed the peaks at 1655 and $1550 \mathrm{~cm}^{-1}$, corresponding to the bending mode of the free $\mathrm{NH}_{2}$ groups, the $\mathrm{CH}_{x}$ asymmetric/ $\mathrm{CH}_{2}$ deformations at 2925, 1368 and $780 \mathrm{~cm}^{-1}$, a peak of $\mathrm{Si}-\mathrm{O}-\mathrm{Si}$ stretching mode due to Piranha treatment for $\mathrm{SiH}$ and thermal oxidation for $\mathrm{SiO}_{2}$ at 1090-1010 $\mathrm{cm}^{-1}$. In the case of the $\mathrm{SiH}$ sample, the characteristic peak of backbone $\mathrm{Si}-\mathrm{H}$ bonds at $2100 \mathrm{~cm}^{-1}$ was also observed. FTIR characterization cannot show structural differences between APTES and APDMES: the two compounds have the same alkyl chain but while APTES has three ethoxy groups, that cause polymerization of compound and thus a better coverage of surface, APDMES instead has two
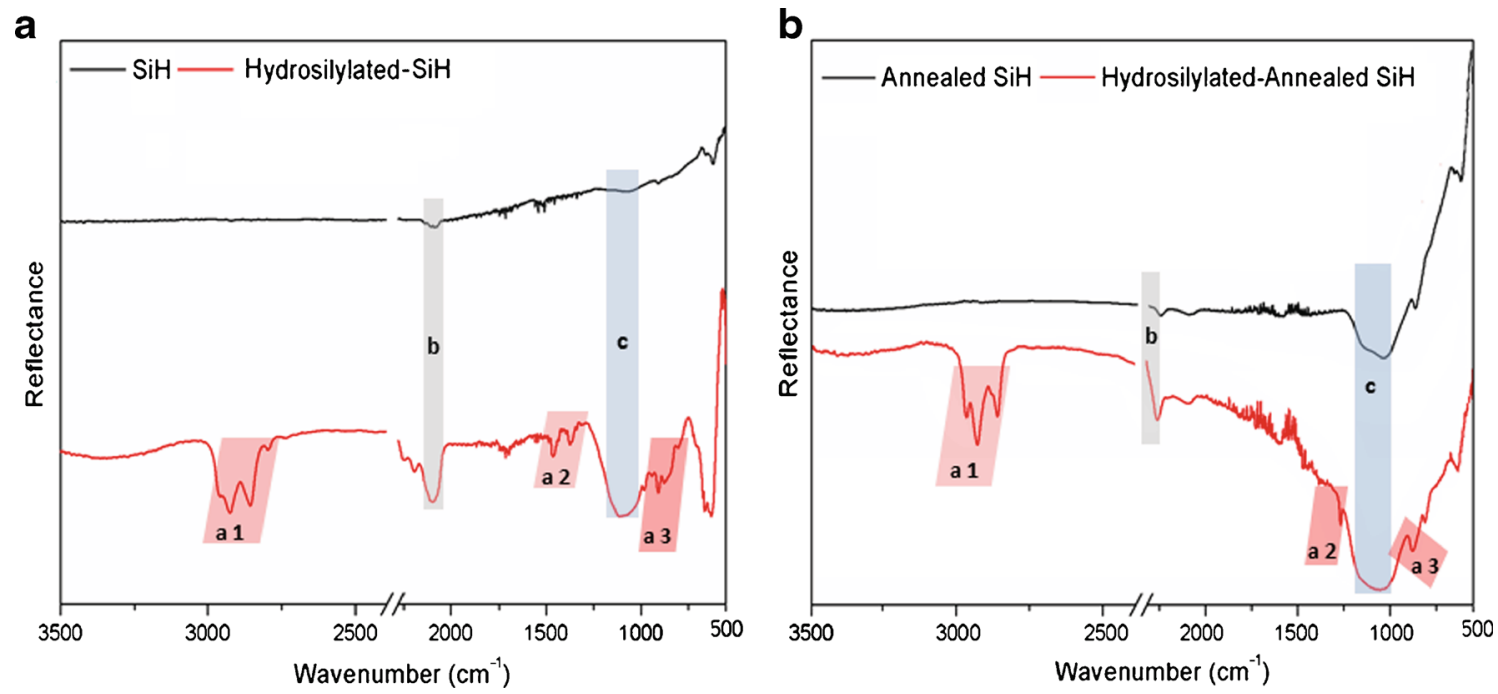

Fig. 2 FTIR spectra of pSi powders (BS419) before and after the hydrosilylation (a) and after annealing and hydrosilylation (b) 
a

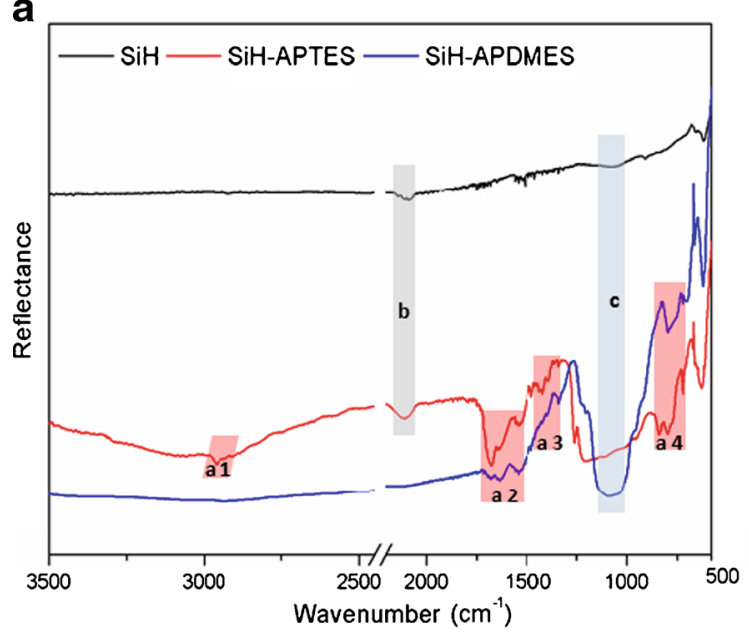

b

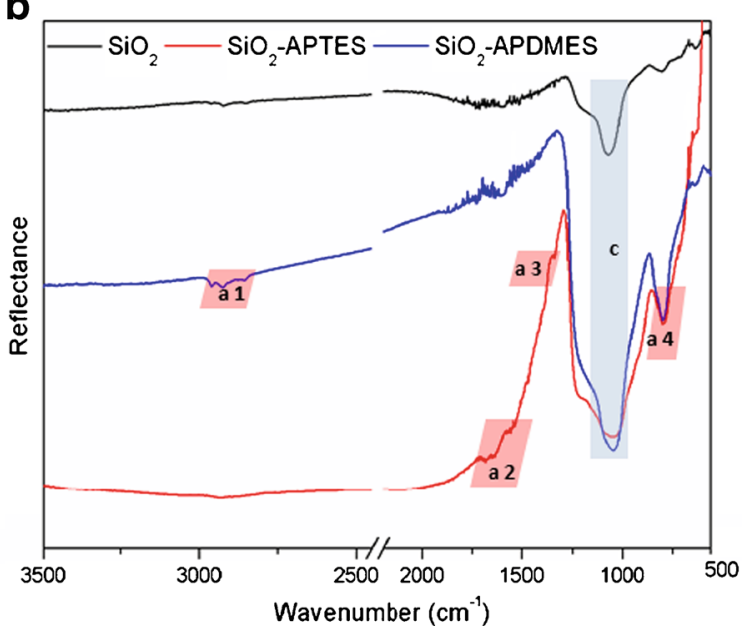

Fig. 3 FTIR spectra of porous silicon BS419 (a) and oxidized porous silicon BS445 (b) powders before and after the silanization with APTES and APDMES

methyl groups and one ethoxy group and does not polymerize. The DLS measurements confirmed the correct functionalization of particles surface due to a change of sample zeta potential from $-16( \pm 4) \mathrm{mV}$ to $+7( \pm 3) \mathrm{mV}$ after silanization.

\subsubsection{HPLC Based Chemical Reactivity Assay}

When incubated with $20 \mathrm{mg}$ of as-etched $\mathrm{pSi}$, the Lansoprazole-methanol solution showed a maximum degradation of $30 \%$ compared with a $20 \mathrm{mg}$ sample of commer-

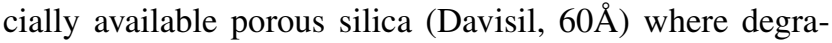
dation is less than $0.02 \%$ (Fig. 4). Previous results have shown that when the Lansoprazole-methanol solution was incubated with samples of thermally passivated $\mathrm{pSi}$, the proportion of Lansoprazole degradation was seen to decrease as the temperature used to passivate the silicon skeleton was increased over the range $500-800{ }^{\circ} \mathrm{C}$ [49].

Table 3 FTIR peak values of porous silicon/silica powders after silanization

\begin{tabular}{|l|}
\hline \multicolumn{1}{|c|}{ SiH-APTES/APDMES } \\
SiO $_{2}$-APTES/APDMES \\
\hline a $1=2925 \mathrm{~cm}^{-1} \mathrm{CHx}$ asymmetric stretch \\
\hline a $2=1368 \mathrm{~cm}^{-1} \mathrm{CHx}$ symmetric stretch \\
\hline a $3=1655-1550 \mathrm{~cm}^{-1} \mathrm{NH}$ bend \\
\hline a $4=1368-780 \mathrm{~cm}^{-1} \mathrm{CH}$ stretch \\
\hline b $=2100 \mathrm{~cm}^{-1} \mathrm{Si}_{-1} \mathrm{H}^{-}$stretch \\
\hline c $=1090-1100 \mathrm{~cm}^{-1}$ Si-O-Si stretch \\
\hline
\end{tabular}

The Lansoprazole mediated degradation of oxidepassivated and derivatized samples is shown in Table 4. Biosilicon material (BS445) that was passivated at $400{ }^{\circ} \mathrm{C}$ and annealed in the presence of nitrogen was found to be highly reactive. This batch showed almost complete degradation (95\%) of Lansoprazole; more than $65 \%$ than the as-anodized control batch which degrades Lansoprazole by 28-30\%. The hydrosilylation of anodised batches reduced degradation significantly to $12.32 \%$ whereas hydrosilylation and annealing further reduced it to $3.95 \%$. Derivatization of anodised batch with APDMES resulted in a slight decrease in degradation, while with APTES the reactivity was seen to lower significantly $(0.58 \%)$. pSi samples (BS419) which have been oxide-passivated at $700{ }^{\circ} \mathrm{C}$ for more than 16 hours and then derivatized showed low reactivity values of $<1 \%$.

Table 4 shows HPLC measurement of surface reactivity of porous silicon powders (BS419 \& BS445) after varied treatments.

\subsection{Dye Based Reactivity Assay}

A fluorimetric dye method was used for measuring the reactivity of porous silicon samples. Chemical reactivity of porous silicon has been ascribed to hydrides associated with the surfaces of the particles $(12,21)$. These hydride surfaces reduce Resazurin (blue), a marginally fluorescent dye, to pink Resorufin which is highly fluorescent.

A linear relationship between Resorufin concentration and fluorescence was seen over the concentration range from $0.07-1.68 \mu \mathrm{g} / \mathrm{mL}$ with an R2 value of 0.9965 (Fig. 5). This standard plot was used to quantify the amount of Resazurin converted to Resorufin due to the presence of hydride bonds at the surface. The method was validated 
Fig. 4 Chromatograms of (a) free Lansoprazole and Lansoprazole following incubation with (b) commercial sol-gel $\mathrm{SiO}_{2}$ (c) unpassivated porous silicon
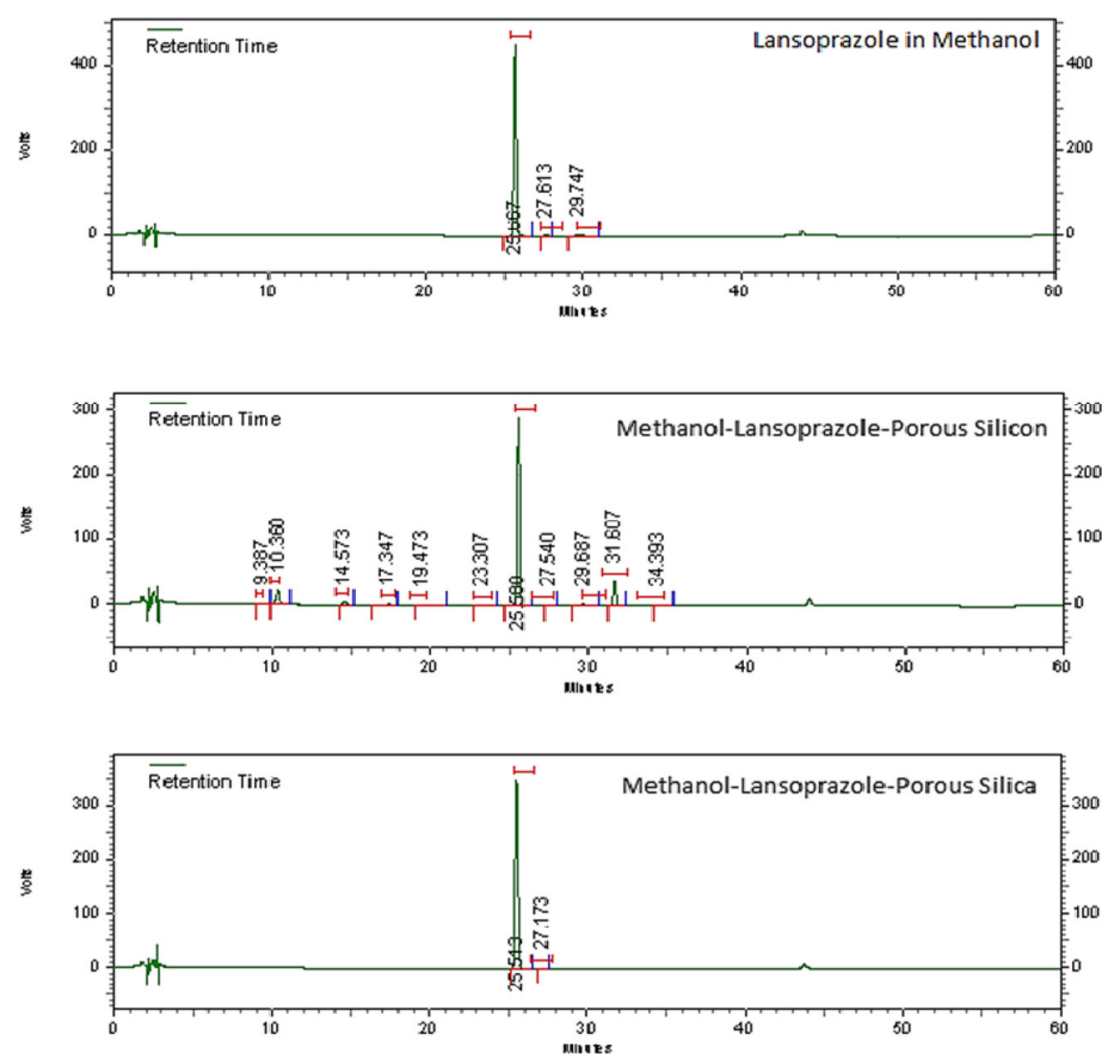

for accuracy, linearity and specificity. Potential interference from the derivatization reagents (APTES, APDMES and decene) on the fluorescence assay was found to be negligible or within the acceptable limits of a fluorimetric assay for all three reagents (Table 5).

The amount of Resorufin produced can be used as a direct measurement of the chemical reactivity of the sample. As-anodized or native oxide passivated $\mathrm{pSi}$ samples cannot be analysed by this assay due to poor wettability; therefore slightly passivated controls $\left(500{ }^{\circ} \mathrm{C}\right.$ for 1 hour) are used as a positive control in this assay. When $20 \mathrm{mg}$ of this form of $\mathrm{pSi}$ was incubated with $1 \mathrm{ml}$ of $(2.5 \mu \mathrm{g} / \mathrm{ml})$ Resazurin, approximately $2.2-2.4 \mu \mathrm{g} / \mathrm{mL}$ of
Resorufin was measured. Incubation with porous silica on other hand generates only $0.01 \mu \mathrm{g} / \mathrm{mL}$ of Resorufin under similar conditions. The dye based data on reactivity of $\mathrm{pSi}$ samples are shown in Table 5. With hydrosilylated pSi samples (as with HPLC) it was noted that APDMES derivatized samples showed higher reactivity compared with derivatization by APTES. While hydrosilylation alone did not reduce the reactivity significantly, annealing and hydrosilylation proved to reduce the hydride content more effectively. Oxidized batches showed significantly lower reactivity after hydrosilylation with APDMES and the improvement is even more pronounced after hydrosilylation with APTES.
Table 4 HPLC measurement of surface reactivity of porous silicon powders (BS419 \& BS445) after varied treatments

\begin{tabular}{lll}
\hline Sample & $\begin{array}{l}\text { Chemical passivation } \\
\text { treatments }\end{array}$ & $\begin{array}{l}\text { Degradation of Lansoprazole } \\
\text { post incubation }(\%)\end{array}$ \\
\hline BS445 & Si-H annealed $\left(400^{\circ} \mathrm{C}\right.$ in nitrogen) & 94.9 \\
BS445 & Si-H \& APDMES & 16.51 \\
BS445 & Si-H \& hydrosilylation & 12.32 \\
BS445 & Si H-annealed \& hydrosilylation & 3.95 \\
BS419 & Si-H \& oxidation \& APDMES & 0.64 \\
BS445 & Si-H \& APTES & 0.58 \\
BS419 & Si-H \& oxidation & 0.54 \\
BS419 & Si-H \& oxidation \& APTES & 0.30 \\
& Sol gel silica (control) & 0.01 \\
\hline
\end{tabular}




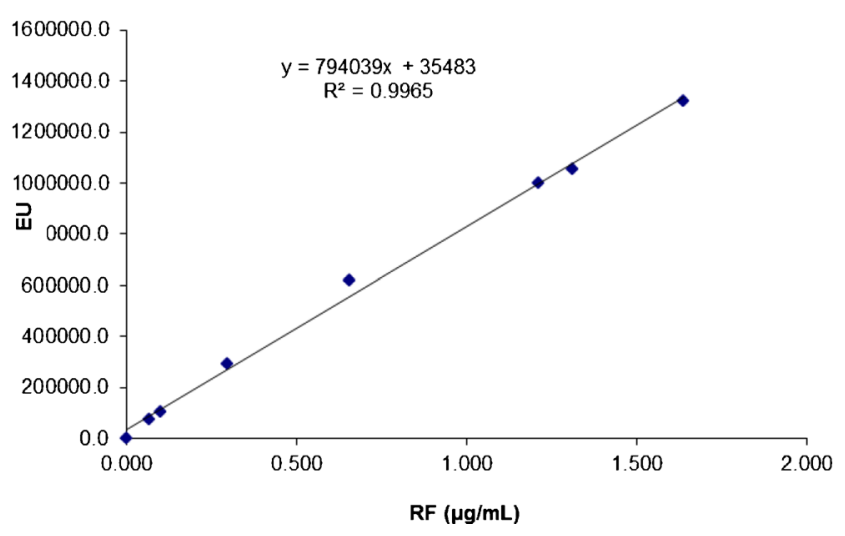

Fig. 5 Relationship of Fluorescence (y-axis) to Resorufin Concentration (x-axis)

\subsubsection{Chemography Results}

As-etched pSi can generate darkening on a photographic plate within 10 minutes exposure (28). When as-etched $\mathrm{pSi}$ and $800{ }^{\circ} \mathrm{C}$ passivated (rotary furnace, 16 hours) pSi were exposed for 4 hours at $100 \%$ humidity, only as-etched $\mathrm{pSi}$ showed a dark spot while the oxidized sample showed no image, indicating that the rate of silane emission from this sample is much slower or negligible (Fig 6). While hydrosilylated samples showed a strong image after 20 hours, pSi treated with APTES and APDMES showed a faint image after 20 hours which was stronger after 72 to 100 hours. Samples which were passivated for $700{ }^{\circ} \mathrm{C}$ for 16 hours showed a faint image after 100 hours, which was completely absent after additional hydrosilylation with APTES or APDMES.

Figure 6 provides an example of the chemographic effects seen and summarized in Table 6. Figure 6a shows the image of powders before exposing them to photographic film and Fig. $6 \mathrm{~b}$ shows a strong image seen from the Si$\mathrm{H}$ hydrosilylated sample; a faint image seen from the $\mathrm{Si}-\mathrm{H}$ APDMES sample; and no image seen from Si-H \& APTES and $\mathrm{Si}-\mathrm{H}$ annealed samples.

Table 5 Dye reactivity of porous silicon powders (BS419 \& BS445) after varied treatments

\begin{tabular}{ll}
\hline Chemical passivation treatments & $\begin{array}{l}\text { Dye reactivity/Resorufin } \\
\text { Concentration }(\mu \mathrm{g} / \mathrm{mL})\end{array}$ \\
\hline Si-H \& APDMES & 1.28 \\
Si-H \& hydrosilylation & 1.13 \\
Si-H \& oxidation & 1.00 \\
Si-H \& APTES & 0.79 \\
Si H-annealed \& hydrosilylation & 0.68 \\
Si-H \& oxidation \& APDMES & 0.18 \\
Si-H \& oxidation \& APTES & 0.02 \\
Sol gel silica (control) & 0.02
\end{tabular}
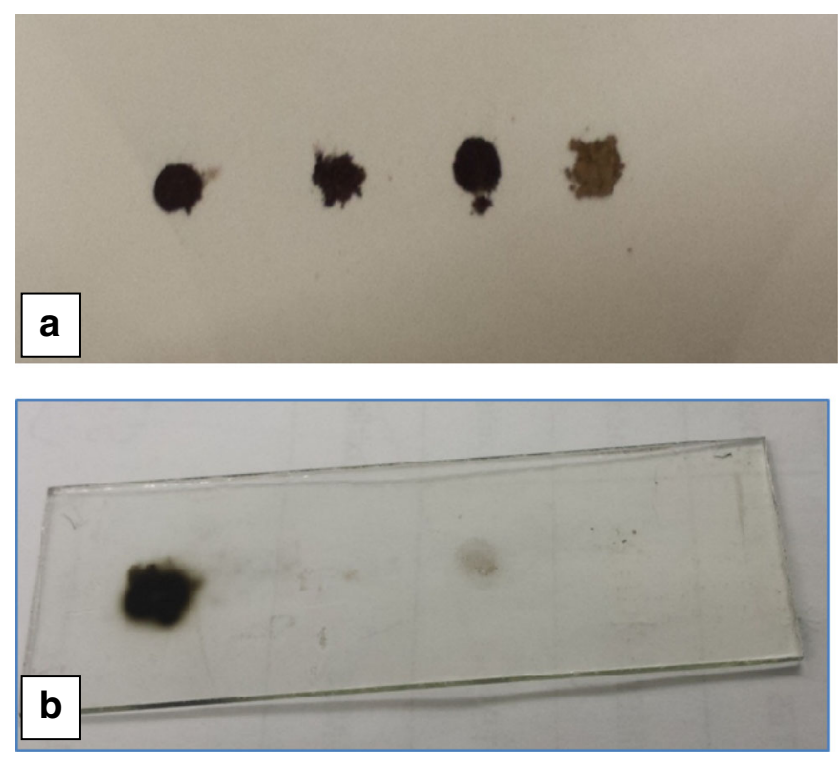

Fig. 6 a photograph b chemograph of four samples after 20 hour exposure. (From left to right: Si-H hydrosilylated; Si-H annealed in nitrogen; Si-H APDMES; Si-H APTES)

\section{Discussion}

Residual chemical reactivity of oxidized porous silicon, as quantified by these specific assays and compared with amorphous silica, could in principle arise from a number of sources that we now discuss in turn. Topics addressed are silicon-hydride bond removal and its re-formation, trace silane emission, trace etch residues, trace solvents, trace metal contamination and the $\mathrm{pH}$ microclimate within the pores.

Residual silicon-hydride bonding arrangements are the primary candidate for residual chemical reactivity. Stoichiometric amorphous silica can be rendered more reactive by the introduction of such hydride bonding [31, 32]. Derivatization of porous silicon, by introduction of silicon-carbon bonding, does not remove all such hydride [12, 33], even when side-reactions are minimized [34]. This is generally ascribed to steric hindrance effects [12] but could also be due to some silicon-hydride bonding being within the silicon skeleton, as recently proposed [35]. In this regard, thermal dehydrogenation should be much more effective at removing all silicon-hydride, both surface and internal species. Due to its thermal stability, surface monohydride is reported to require temperatures above $550{ }^{\circ} \mathrm{C}$ for its removal. [36] Hydrogen trapped internally by point defects within the skeleton could need temperatures as high as 700 ${ }^{\circ} \mathrm{C}$ [37]. For this reason, samples were oxidized at $700{ }^{\circ} \mathrm{C}$ for a long duration and, at least by FTIR, silicon-hydride was reduced to undetectable levels (see Fig. 3b). However, even if silicon-hydride is completely thermally removed, the interaction of residual elemental silicon in the porous 
Table 6 Chemography of porous silicon powders (BS419 \& BS445) after varied treatments

\begin{tabular}{ll}
\hline Chemical passivation treatments & Chemography results \\
\hline Si-H \& hydrosilylation & strong image after 20 hours \\
Si-H \& APTES & faint image after 20 hours; strong image after 72 hours \\
Si H \& APDMES & faint image after 20 hours; stronger image after 100 hrs \\
Si H- annealed \& hydrosilylation & very faint image after 100 hours exposure \\
Si H , oxidation and APDMES & no image after100 hours exposure \\
Si H, oxidation and APTES & no image after100 hours exposure \\
Sol gel silica (control) & no image after 100 hours exposure \\
\hline
\end{tabular}

structure with aqueous environments can lead to its reintroduction. Convincing evidence for silicon-hydride creation from silicon surfaces during alkali etching [38], tribochemical etching in water $[39,40]$ and storage in weakly alkali solutions like body fluids [41] is available in the literature.

Gaseous silane emission is a second potential source of reactivity [7], especially for biological molecules stored for long periods within the mesoporous structure. Evidence for trace silane emission from both silicon and porous silicon comes from chemography studies [20, 42], FTIR [42, 43] and mass spectrometry [44]. Silane, like surface siliconhydride bonding, is a potential reducing agent that could react with the two molecular probes used in these assays. Tests where the dye solution was indirectly exposed to silane gas emission from unpassivated porous silicon however were inconclusive, possibly due to its rapid hydrolysis by water during transit [Shabir Q, Loni A. unpublished data]. For the removal of silicon trihydride groups thought to generate monosilane, thermal treatments should be very effective; this is in accord with the nitrogen annealing treatments having a pronounced effect with regard to silane emission (no chemographic image in Fig. 6) but residual monohydride content (residual activity) in both drug assay (Table 4) and dye assay (Table 5).

Trace etch residues, such as silicon tetrafluoride and hexafluorosilicic acid, could potentially introduce chemical reactivity [22]; however, rigorous water and solvent rinsing with multiple exchanges was employed in sample preparation. Fluoride levels in selected batches were undetectable by XRF and XPS [Loni A. unpublished data 45] but the sensitivity of these techniques for this element is not particularly high. Trace solvent levels in selected batches were also quantified and showed ethanol/methanol content at pharmacopeia-acceptable ppm levels (see Table 1).

The primary trace metal contaminants detected were zirconium $\sim 1000 \mathrm{ppm}$ (from the zirconia ball milling process), copper (17-22 ppm) and nickel (11-12 ppm). Arsenic, cadmium, mercury, lead, aluminum, barium, chromium, tungsten, molybdenum, platinum and manganese were all below the detection limit of XRF (Table 1). The presence of zirconium has potential relevance to the final topic discussed next; the surface acidity of oxidized porous silicon.
The $\mathrm{pH}$ assays demonstrated that oxidized porous silicon microparticles had lower $\mathrm{pH}$ values than commercial silicas prepared by sol-gel technology (e.g. pH 5-7) but comparable values to pharmacopeia-grade fumed silicas like Cab-O-Sil ( $\mathrm{pH}$ 3.6-4.5). The monomer degradation product, orthosilicic acid, is weakly acidic with a pKa of 9.9 [45]. Polysilicic acids that can arise from hydrolysis-induced supersaturation are stronger acids than the monomer, with $\mathrm{pKa}$ values up to 6.5 [45]. One minor contaminant detected in oxidized porous silicon that could influence microclimate $\mathrm{pH}$ is zirconium from the ball milling process. Although zirconia is an established biomaterial [46], the incorporation of zirconium into silica is known to increase catalytic activity via Lewis site acidity [47]. Subsequent preliminary tests have shown the drug-based HPLC assay to be strongly $\mathrm{pH}$ sensitive, but the dye-based fluorometric assay less so [Webb K, Shabir Q. unpublished data]. Nevertheless all three assays appear to have value in optimizing porous silicon passivation for extended dry and wet storage API stability testing.

Biodegradable porous silicon is currently under assessment as a biomaterial for a wide range of therapeutic medical applications, including brachytherapy, chemotherapy, theranostics and tissue engineering [48]. Its in-vivo biocompatibility is the subject of an increasing number of studies [49] but in-vitro pharmacopeia-grade testing is also essential for translation of those applications requiring drug delivery into clinical evaluation. We have provided some assays and discussed some issues that should be helpful in this regard.

\section{Conclusions}

When minimizing the chemical reactivity of porous silicon for drug delivery applications, attention needs to be given to complete removal of silicon hydride bonding, effective rinsing and drying techniques that remove electrolyte components and etch residues, trace metal contamination arising from microparticle batch manufacture and the $\mathrm{pH}$ microclimate within the pores. Drug and dye-based storage assays have been developed to quantify residual reactivity. Chemography is currently only a qualitative assay, 
but results appear to broadly correlate with the molecular assays. Further research continues to elucidate the causes of residual chemical reactivity, as monitored by these sensitive assays, and the extent to which the data correlates with model API stability during lengthy storage.

Open Access This article is distributed under the terms of the Creative Commons Attribution 4.0 International License (http:// creativecommons.org/licenses/by/4.0/), which permits unrestricted use, distribution, and reproduction in any medium, provided you give appropriate credit to the original author(s) and the source, provide a link to the Creative Commons license, and indicate if changes were made.

\section{References}

1. Prestidge CA, Barnes TJ, Lau CH, Barnett C, Loni A, Canham LT (2007) Mesoporous silicon : a platform for the delivery of therapeutics. Expert Opin Drug Deliv 4(2):101-110

2. Salonen J, Kaukonen AM, Hirvonen J, Lehto VP (2008) Mesoporous silicon in drug delivery applications. J Pharm Sci 97(2):632-653

3. Anglin EJ, Cheng L, Freeman WR, Sailor MJ (2008) Porous silicon in drug delivery devices and materials. Adv Drug Deliv 60(11):1266-1277

4. Shahbazi MA, Herranz B, Santos HA (2012) Nanostructured porous Si-based nanoparticles for targeted drug delivery. Biomatter 2(4):296-312

5. Barnes TJ, Jarvis KL, Prestidge CA (2013) Recent advances in porous silicon technology for drug delivery. Ther Deliv 4(7):811823

6. Martín-Palma RJ1, Hernández-Montelongo J, Torres-Costa V, Manso-Silván M, Muñoz-Noval Á (2014) Nanostructured porous silicon-mediated drug delivery. Expert Opin Drug Deliv 11(8):1273-1283

7. Canham LT (1995) Bioactive silicon fabrication via nanoetching techniques. Adv Mater 7:1033

8. Park JH, Gu L, Von Maltzahn G, Ruoslahti E, Bhatia SN, Sailor MJ (2009) Biodegradable luminescent porous silicon nanoparticles for in-vivo applications. Nat Mater 8(4):331-336

9. Shabir Q (2014) Biodegradability of porous silicon. In: Canham L (ed) Handbook of Porous Silicon. Springer, Switzerland, pp 235-236

10. Khokhlov A, Valiullin R. (2014) Mesoporous silicon. In: Canham L (ed) Handbook of Porous Silicon. Springer, Switzerland, pp 123-145

11. Wan Y, Apostolou S, Dronov R, Kuss B, Voelcker NH (2014) Cancer-targeting siRNA delivery from porous silicon nanoparticles. Nanomedicine 9(15):2309-2321

12. Sailor MJ (2014) Chemical reactivity and surface chemistry of porous silicon. In: Canham L (ed) Handbook of Porous Silicon. Springer, Switzerland, pp 355-380

13. De Stefano L, Oliviero G, Amato J, Borbone N, Piccialli G, Mayol 1, Rendina I, Terracciano M, Rea I (2013) Aminosilane functionalization of mesoporous oxidized silicon for oligonucleotide synthesis and detection. J Royal Soc Interface 10:20130160

14. Nadarassan DK, Loni A, Shabir Q, Kelly C, O’Brien H, Caffull E, Webb K, Canham LT, Maniruzamman M, Trivedi V, Douroumis D (2015) Ultrahigh drug loading and release from biodegradable porous silicon aerocrystals. Extended Abstract No. $82542^{\text {nd }}$ Meeting of Controlled Release Society. 26-29 ${ }^{\text {th }}$ July Edinburgh, UK
15. Estey T, Kang J, Schwendeman SP, Carpenter JF (2006) BSA degradation under acidic conditions: a model for protein instability during release from PLGA delivery systems. J Pharm Sci 95(7):1626-1639

16. Agrawali CM, Athanasiou KA (1997) Technique to control $\mathrm{pH}$ in vicinity of biodegrading PLA-PLGA implants. J Biomed Mater Res 38(2):105-114

17. Kuhlmann J, Bartsch I, Willbold E, Schuchardt S, Holz O, Hort N, Höche D, Heineman WR, Witte F (2013) Fast escape of hydrogen from gas cavities around corroding magnesium implants. Acta Biomater 9(10):8714-8721

18. Song GL, Song SZ (2006) Corrosion behaviour of pure magnesium in a simulated body fluid. Acta Phys Chim Sin 22(10):122-126

19. Emmanuel B, Thomas E, Annette B, Gabriele L, Helmut L, Heinrich W (2005) Reference values for serum silicon in adults. Anal Biochem 337:130-135

20. Jay T, Canham LT, Heald K, Reeves CL, Downing R (2000) Autoclaving of porous silicon within a hospital environment: potential benefits and problems. Phys Stat Solidi A 182(1):555-560

21. Riikonen J, Salomäki M, van Wonderen J, Kemell M, Xu W, Korhonen O, Ritala M, MacMillan F, Salonen J, Lehto VP (2012) Surface chemistry reactivity and pore structure of porous silicon oxidized by various methods. Langmuir 28:10573-10583

22. Koynov S, Pereira RN, Crnolatac I, Kovalev D, Huygens A, Chirnovy V, Stuzmann M, de Witte P (2011) Purification of nano-porous silicon for biomedical applications. Adv Engn Mater 13(6):B225-B233

23. Loni A (2014) Porous silicon formation by anodization. In: Canham L (ed) Handbook of Porous Silicon. Springer, Switzerland, pp 11-22

24. Terracciano M, Rea I, De Stefano L, Rendina I, Oliviero G, Nici FD, Errico S, Piccialli G, Borbone N (2014) Synthesis of mixed sequence oligonucleotides on mesoporous silicon: chemical strategies and material stability. Nanoscale Res Lett 9:317

25. Krishnamohan T, Ialitha G, Sharma RV, Sambasivarao L, Babu JM, Younus M, Raju AS, Murthy YLN (2012) Method development and validation for estimation of related compounds present in lansoprazole bulk drug by ultra high pressure liquid chromatography. Asian J Res Chem 5(7):859-865

26. O'Brien J, Wilson I, Orton T, Pognan F (2000) Investigation of the Alamar Blue (resazurin) fluorescent dye for the assessment of mammalian cell cytotoxicity. Eur J Biochem 267:5421-5426

27. Low SP, Williams KA, Canham LT, Voelcker NH (2006) Evaluation of mammalian cell adhesion on surface-modified porous silicon. Biomaterials 27:4538-4546

28. Canham LT, Saunders SJ, Healey PB, Keir AM, Cox T1 (1994) Rapid chemography of porous silicon undergoing hydrolysis. Adv Mater 6(11):865-868

29. Buriak JM, Stewart MP, Geders TW, Allen MJ, Choi HC, Smith JD, Raftery D, Canham LT (1999) Lewis acid mediated hydrosilylation on porous silicon surfaces. J Am Chem Soc 121(49):1149111502

30. Lansoprazole Monograph (2006) United States Pharmacopeia. USP 29 NF24: 1229

31. Vella E, Buscarino G, Boscaino R (2011) Structural organization of silanol and silicon hydride groups in the amorphous silicon dioxide network. Eur Phys J B 83:47-52

32. Claudio M, Manfred JDL (1969) Reactive Silica II The Nature of the Surface Silicon Hydrides. J Phys Chem 73(2):327-333

33. Huck LA, Buriak JM (2014) Silicon-carbon bond formation on porous silicon. In: Canham L (ed) Handbook of Porous Silicon. Springer, Switzerland, pp 683-693

34. Boukherroub R, Petit A, Loupy A, Chazalviel JN, Ozanam F (2003) Microwave -assisted chemical functionalization of hydrogen-terminated porous silicon surfaces. J Phys Chem 107(48):13459-13462 
35. Loni A, Canham LT (2013) Exothermic phenomena and hazardous gas release during thermal oxidation of mesoporous silicon powders. J Appl Phys 113:173505

36. Gupta P, Colvin VL (1988) George SM.Hydrogen desorption kinetics from monohydride and dihydride species on silicon surfaces. Phys Rev B 37(14):8234-8243

37. Stein HJ (1975) Bonding and thermal stability of implanted hydrogen in silicon. J Electronic Mater 4(1):159-174

38. Wind RA, Jones H, Little MJ (2002) Hines MA OrientationResolved Chemical Kinetics: Using Microfabrication to Unravel the Complicated Chemistry of KOH/Si Etching. J Phys Chem B106(7):1557-1569

39. Mizuhara K, Hsu SM Tribochemical reaction of oxygen and water on silicon surfaces. In: Dowson D (ed) Wear Particles.1992; Elsevier Science Publishers B. V. p 323

40. Muratov VA, Olsen JE, Gallois BM, Fischer TE, Bean JC (1998) Tribochemical Reactions of Silicon: An in Situ Infrared Spectroscopy Characterization. J Electrochem Soc 145(7):2465-2470

41. Ulin V, Ulin N, Soldatenkov F, Semenov A, Bobyl A (2014) Surface of porous silicon under hydrophilization and hydrolytic degradation. Semiconductors 48(9):1211-1216
42. Lampert I, Fußstetter H, Jacob H (1989) Evidence for SiH4 Formation during the Reaction of Water with a Silicon Surface. J Electrochem Soc 133(7):1472-1474

43. Salonen J, Lehto VP, Laine E (1997) The room temperature oxidation of porous silicon. Appl Surf Sci, 191-198

44. Gunasingham PV, Goldspink G (2000) An investigation into silane evolution from porous silicon by temperature programmed desorption method. J Porous Mater 7:187-190

45. Iler RK (1979) The Chemistry of Silica: solubility, polymerization, colloid and surface properties and biochemistry. Wiley, New York

46. Piconi C, Maccauro G (1999) Zirconia as a ceramic biomaterial. Biomaterials 20:1-25

47. Rodríguez-Castellóna E, Jiménez-Lópeza A, Maireles-Torresa P, Jonesb PD, Rozièreb J, Trombettac M, Buscac G, Lenardad M, Storarod L (2003) Textural and structural properties and surface acidity characterisation of mesoporous silica-zirconia molecular sieves. J Solid State Chem 175:159-169

48. Santos H (ed.) (2014) Biomedical Applications of Porous Silicon, Woodhead Publishing

49. Low SP, Voelcker NH Biocompatibility of porous silicon. In: Canham L (ed) Handbook of Porous Silicon, pp 381-393 